
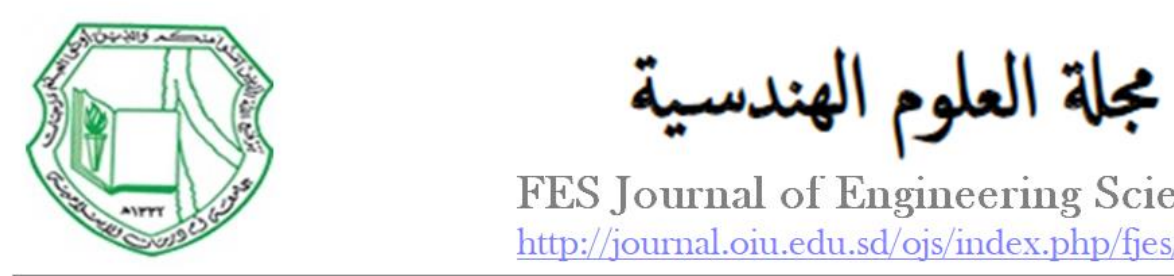

FES Journal of Engineering Sciences

http://journal.oiu.edu.sd/ojs/index.php/fjes/index

\title{
A Novel Walking Speed Control Strategy for Power Augmentation Exoskeletons based on Neural Networks
}

\author{
Abusabah I. A. Ahmed ${ }^{*}$ and Mohammed H. H. Musa² \\ 1 Electrical Engineering Department, Karary University, Omdurman, Sudan \\ 2 College of Engineering, Almughtarbeen University, Khartoum, Sudan \\ * Corresponding author: Abusabah I. A. Ahmed (abusabah22@hotmail.com). \\ Article history: Received 26 March 2020, Received in revised form 26 April 2020, Accepted 20 October 2020
}

\begin{abstract}
This paper addresses a novel sensing technique to minimize the interaction force of walking speed transitions during the navigation of a coupled human-exoskeleton power augmentation system. The proposed technique is able to classify the intended walking speed based on Dual Reaction Force (DRF) Sensor. The human brain as a complex information processing device is quite difficult to be simulated, especially when consider the processing speed and the speed of sensed signals through the human nervous system throughout the human body. We developed the DRF sensors for preemptive identification of pilot intentions for walking speed transitions to augment the response of the exoskeleton to shadow pilot's movements. The Dynamic Threshold Neural Networks (DTNNs) is used to classify the input signals and make a decision on the transition of system's walking speed according to the pilot's intentions and walking speed limitation. The actions for walking speed transitions are simulated in MATLAB/Simulink, and Variable Admittance Controller (VAC) and applied. To show the efficiency of the proposed walking speed control strategy, comparison is conducted with ordinary VAC algorithm technique.
\end{abstract}

Keywords: Power Augmentation Exoskeleton, Dynamic Threshold Neural Networks (DTNNs), Dual Reaction Force (DRF) Sensors, Walking Speed Transition, Variable Admittance Control.

\section{INTRODUCTION}

The motion control of power augmentation exoskeletons have gained considerable interests in recent years, especially in hazardous places and applications. Nowadays, the lower limb exoskeleton system is designed for human power augmentation, load transfer and endurance increasing applied a variety of motion control methodologies [1]-[4].

Sensitivity Amplification Control (SAC) algorithm was proposed for Berkeley Lower Extremity EXoskeleton (BLEEX) control which aimed to reduce the sensors complexity and used the interaction force measured in the exoskeleton as main input [5], [6]. SAC needs no direct measurements from the pilot or the human-machine interface (e.g. no force sensors between them); instead, the controller estimates, based on measurements from the exoskeleton suits only. The cost of the control process in this strategy is extremely high, in both hardware and software, the complicated systems for signals conditioning and useful feedback extraction make heavy calculations. The high level of sensitivity in response to the forces and torques imposed by the pilot is counted as negative time and resource consuming process. Human Intention Estimators (HIE) are implemented for Hybrid Assistive Limb (HAL) control by calculating the reference patterns of the pilot by measuring human-exoskeleton interaction directly from Electromyography signals (EMG) [7], [8]. The Japanese-built, wearable, HAL exoskeleton, for example, offers improved endurance and strength for users but is not mind-controlled. Instead of brain waves, HAL picks up EMG signals, the electrical pulses 
generated by the muscles and uses these to send commands to the exoskeleton. It also partially relies on the users existing mobility and balance to operate. Meanwhile, Berkeley Bionics in the US has developed an EMG-based "medical exoskeleton" that enables people with reduced mobility and strength to walk upright. However, it requires a supporting device to keep users balanced. HAL uses electrical signals sent to the muscles from the brain to anticipate the wearer's movement. The Variable Admittance Control (VAC) algorithm, which applied for the coupled human-exoskeleton system control is meant by developing in this work [4].

In this paper, we developed a novel walking speed control strategy based on Feed forward Neural Networks (FNNs) for preemptive identification of the pilot intentions for walking speed transitions. The novel method learns the walking speed changing factor on-line through the new presented Dual Reaction Force (DRF) sensors system. Single hidden layer FNNs (SFNNs) can reflect the pilot intentions for walking speed transitions based on the presented sensory system and Adaptive Central Patterns Generators (ACPGs).

The new developed auxiliary tools like DRF and additional applications make a big difference in overall performance of proposed strategy comparing to SAC and HAL. The DTNNs and ACPGs provide improved reliability over similar strategies reflected in calculation time minimization of on-line controllers. Cost-effective intension identification system applied in coupled human exoskeleton systems motion control for the reliable and accurate intentions identification. The proposed system can perform well and make a preemptive intention identification of maneuverable exoskeleton systems.

Applying the proposed control algorithm, distributed control system was embedded in the HUALEX exoskeleton system. The distributed control system is constructed with the main controller and four node controllers. The main controller is set at the backpack, which running the control algorithm. Nearby each active joint, a node controller is installed for two purposes:
1. Collecting sensor data for the main controller;

2. Executing commands from the main controller.

For ensuring the real-time performance of control algorithm, node controllers communicate with the main controllers via Controller Area Network (CAN). HUALEX has total three kinds of sensors for its current state obtaining. Encoders are integrated into joint actuators, which measuring the current state of each joint. An accelerometer is set at the backpack for observing the walking velocity of the pilot. The motion control of HUALEX system have perfect performance, high accuracy, minimum tracking error and minimum interaction force applying the new sensory technique.

The paper is organized as follows: Section 2 shows the composition of HUALEX, the integration of the system, sensing terminals and driving system. We show the last work performance illness during gait transitions in Section 3. Section 4 shows the implementation of proposed strategy. The performance analysis of proposed motion control strategy is detailed in section 5. Finally, conclusions and further development are drawn in section 6 .

\section{HUMAN POWER AUGMENTATION LOWER EXOSKELETON (HUALEX) DESCRIPTION}

Totally 10-DoFs revolute joints are adopted for the HUALEX design as shown in Fig.1, two-linkage revolute mechanism regarded as a 2-DOFs multilink pendulum is actuated parallel to the human thigh and shank when

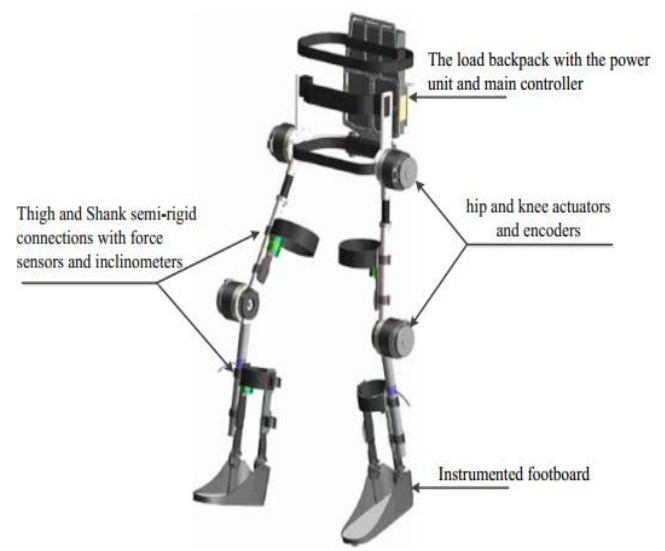

Fig. 1. The prototype of HUman power Augmentation Lower Exoskeleton (HUALEX). 
The exoskeleton is coupled to the pilots. The remaining DoFs are un-actuated and passively driven with in human joints movement. Since the proposed control strategy for gait transition is a model-based control strategy, the dynamic model of HUALEX project must be given.

$$
M(\theta) \ddot{\theta}+C(\theta, \dot{\theta}) \dot{\theta}+G(\theta)=\tau_{E x o}+\tau_{h}
$$

where $\theta$ is the vector of each joint angle, $\tau_{\text {Exo }}$ and $\tau_{h}$ represent the input torques from HUALEX and human wearer respectively, $M(\theta)$ is the inertia matrix and a function of $\theta, C(\theta, \dot{\theta})$ is the Coriolis matrix and a function of $\theta$ and $\dot{\theta}$ and $G(\theta)$ is a vector of gravitational torques. The torque $\tau_{h}$ is changing during system's navigation according to human intentions.

The HUALEX actuated and passively driven Degrees of Freedom (DoFs) are designed to guarantee the shadow of all expected pilot's maneuvers. In the sagittal plane, the designed ranges of motion at the hip, knee and ankle joints are $-45^{\circ}$ to $+45^{\circ}, 0^{\circ}$ to $-135^{\circ}$ and $-30^{\circ}$ to $+30^{\circ}$ respectively. The link lengths are adjustable respect to various pilots.

The new developed Dual Reaction Force (DRF) sensors are the valuable addition to the HUALEX's sensing system which facilitates the application of this strategy.

\section{VARIABLE ADMITTANCE CONTROL (VAC)}

The modifications of Variable Admittance Control (VAC) is needed due to ill effects on control process on the gait transition case, the interaction forces resulted on the measured interaction force must have special treatments for smooth transition [9]. The VAC algorithm attempts to minimize the interaction force during speed transitions of the coupled humanexoskeleton systems, i.e. better tracking performance. VAC strategy applied the difference $\Delta \theta(t)$ resulted from interaction forces to modify the input trajectory to satisfy the pilot's intended maneuvers as in (2) [4].

$$
\theta_{h}^{*}(t)=\theta_{h}(t)+\Delta \theta(t)
$$

The dynamic process simulates the on-line input trajectory correction generally as forceposition controller, to modify the input position (walking trajectory) according to the resulted interaction force (pilot intentions). This process was detailed in our previous work, and can be described by the dynamic visco-elastic admittance parameters as in (3).

$$
\begin{aligned}
& K_{d y n}(t)=K_{0} \delta(t) \\
& B_{d y n}(t)=B_{0} \eta(t)
\end{aligned}
$$

where $K_{0}$ and $B_{0}$ represent the initial stiffness and viscous friction coefficients. Experimentally we estimate the dynamic visco-elastic model parameters from collected data applying Recursive Least Square Estimator (RLSE). The considered model for the estimation of dynamic visco-elastic parameters is depicted in (4):

$\eta B_{0} \Delta \dot{\theta}(t)+\delta K_{0} \Delta \theta(t)=\tau_{i}(t)$

The linearity of the relation in (5) is experimentally proved for acceptable range of interaction force, this range is variable depends on human-exoskeleton system function.

$$
\begin{gathered}
J_{c}\left(\ddot{\theta}_{h}(t)-\ddot{\theta}_{E x o}(t)\right)+B_{c}\left(\dot{\theta}_{h}(t)-\dot{\theta}_{E x o}(t)\right)+ \\
K_{c}\left(\theta_{h}(t)-\theta_{E x o}(t)\right)=J^{T}(t) f_{i}(t)
\end{gathered}
$$

Equation (4) is used for experimental estimation of the dynamic visco-elastic model parameters from collected data applying RLSE. The initial value of unknown parameters vector $\left[\begin{array}{ll}\delta K_{0} & \eta B_{0}\end{array}\right]^{T}$ are $\left[\begin{array}{ll}\delta & \eta\end{array}\right]^{T}=\left[\begin{array}{ll}1 & 1\end{array}\right]^{T}, \quad$ while $\left[\begin{array}{ll}K_{0} & B_{0}\end{array}\right]^{T}$ are variable through navigation process. The collected samples are for 2 different gait cycle contains the transition. The inertia coefficient assumed constant. The changing rate of the interaction force between human wearer and exoskeleton are indicating the future changing of visco-elastic model parameters. The performance of VAC is shown in Fig. 2. 


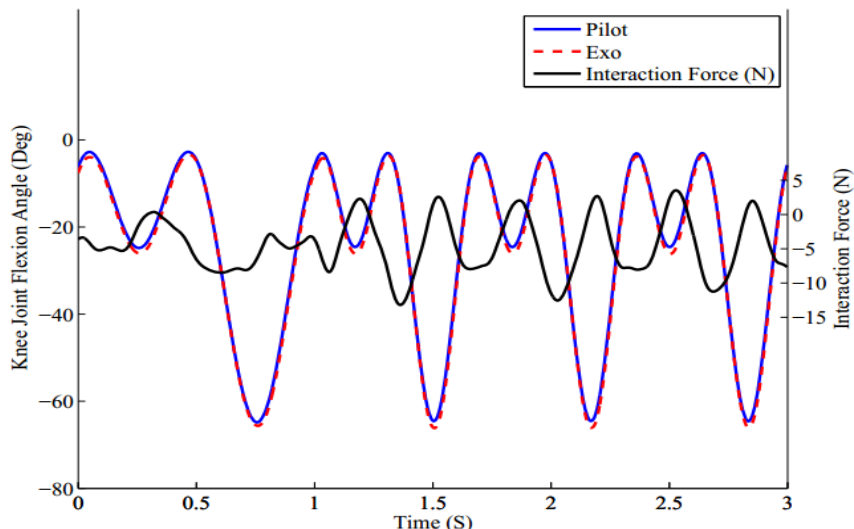

Fig. 2. The performance of Adaptive VAC for walking speed acceleration from $1 \mathrm{~m} / \mathrm{s}(0-1)$ to $1.5 \mathrm{~m} / \mathrm{s}(1-3)$.

\section{PROPOSED MODIFICATION OF VAC}

Many parameters such as step length and stride time are correlated to walking speed, and they can be used to control it [10]. The proposed sensing strategy can control the walking speed of the system using the mismatch in the heel contact time $H_{C T}$ which represents a part of stride length as main feedback. The Adaptive Central Pattern Generators (ACPGs) are used in the input trajectory for the benefit of frequency controllable property. In our case, the teaching signal (input trajectory) is now encoded into a structurally stable limit cycle and it is easy to smoothly control its frequency (gait speed) by changing $\vec{\omega}[11]$.

\section{A. Dual Reaction Force (DRF) sensors}

During human-exoskeleton system navigation, the pilot creates interaction torques that impose on the exoskeleton due to the deviation between the pilot's joint angular position $\theta_{h}$ and that of the exoskeleton $\theta_{E x o}$. If the exoskeleton is able to track the pilot's voluntary motion quickly and simultaneously, the tracking error goes to zero due to the zero interaction (id eal situation). To determine the relationship between walking speed and reaction force mismatch during one gait cycle experimental trails were demonstrated, asking the pilot to speed up and slowdown, the normal speed stance phase duration control is $0.92 \pm 013$, for fast speed is $0.73 \pm 0.06$ as measured for normal subject as in [12]. The applied DRF sensor was investigated in our last work [4], its general configuration as in Fig. 3.
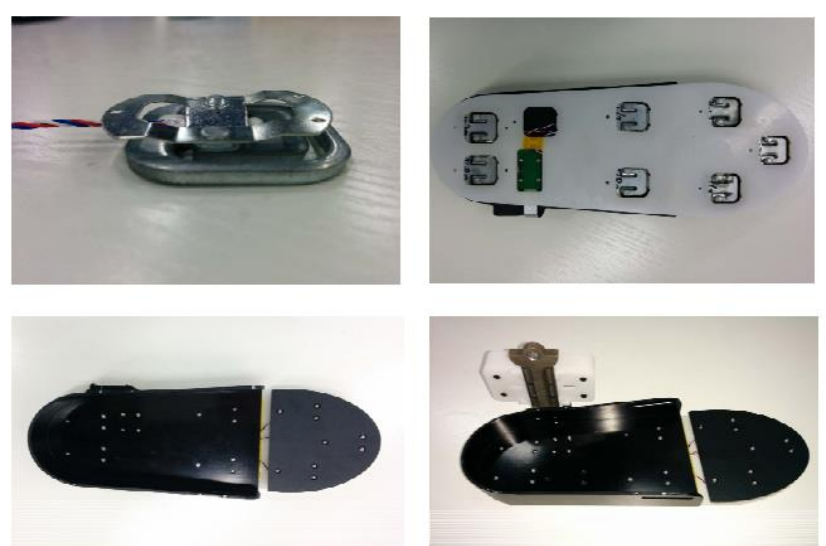

Fig. 3. The Dual Reaction Force (DRF) sensor applied for HUALEX

\section{A. The Fast Recovery Central Pattern Generators (FRCPGs)}

Central pattern generators (CPGs) are biological neural networks that produce rhythmic patterned outputs without sensory feedback (autonomously). CPGs have been shown to produce rhythmic outputs resembling normal even in isolation from motor and sensory feedback from limbs and other muscle targets. CPGs model using Rayleigh oscillators and train this model by human gait oscillation to generate the human like biped locomotion for biped robot successfully achieved [13]. A Voltage Controlled Oscillator (VCO) can be defined as an electronic oscillation frequency is controlled by a voltage input to generate a sinusoidal waveform, relaxation oscillators can generate a saw tooth or triangular waveform according to the:

$f_{t}(t)=f_{q u}+k_{0} v_{i n}(t)$

$\int f_{t}(t) d t=\theta_{\text {out }}(t)$

where $f_{q u}$ is the quiescent frequency of the oscillator, $k_{0}$ is the oscillator sensitivity, or gain, $v_{\text {in }}$ is input voltage, $\theta_{0}$ is the oscillator output. Hopf oscillator is a relatively simple model, and its main advantage is the ability to easily control the oscillator input, the amplitude and frequency of the signal. The CPGs architecture for this application is made of coupled adaptive frequency Hopf oscillators as shown in Fig. 4, which are modified Hopf oscillators that developed by Righetti et al. [14]. 


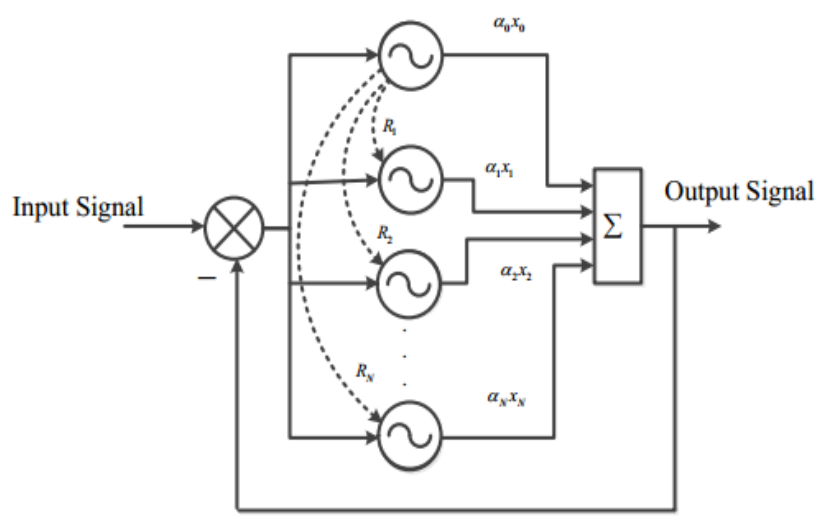

Fig.4. The coupled adaptive frequency Hopf oscillator system for generating CPGs.

\section{DyNAMIC THRESHOLD NeURAL NETWORKS}

In this section for the efficiency augmentation of VAC algorithm considering walking speed transitions, based on the sensory system signals we apply Neural Networks (NNs), to identify the pilot intentions for walking speed changes. Dynamic Threshold Neural networks in which the input signal propagate forward to generate the output successfully applied for classification or prediction tasks [15],[16]. The suitable model for this application is the Feed-forward Single-hidden layer DTNNs, which is often trained by the back propagation algorithm. The designed FSNNs model deals with walking speed changing intentions.

The proposed DTNNs can make on-line predictions of the pilot intended pilot's walking speed dependant on the sensory system outputs and current walking speed. Every time slot $\Delta t$. The hidden layer of the designed DTNNs is the key component because of the neurons special output function which can do major calculations and produce the output. Each neuron takes a set of input data (specified sensors), each is associated with a weight and a numerical value known as bias. The output of each neuron is a function of the output of the weighted sum of each input plus the bias. Let's define the used notations, weights are $\omega_{j, k}^{\ell}$, biases $b_{j}^{\ell}$, and activations $a_{j}^{\ell}$ where $\ell$ is for the layer and $j$ is for the neuron. For the transition control of exoskeleton the VAC algorithm [17] is not enough to ensure tracking error. The mal-performance is due to the considered interaction forces convex during transitions. For this reason, we design the SFNNs controller such that it takes the decision making part on which the VAC algorithm has shown its limitation. The recurrent hidden layer contains one stage which has zero-values weights, the input of non-effective neuron to the next layer.

\section{A. Dynamic threshold function}

Dynamic functions output changes throughout control process depends on the input data set and the current system state. We design dynamic function to control the walking speed of the system within given range. The dynamic parameters of the proposed function reconsider the current walking speed and estimate the suitable threshold to keep the intended walking speed within the allowed range [15].

DTNNs can be applied in learning and feature selection for probabilistic output depends on the system current condition and intended transition. We develop Single hidden layer Feed forward DTNNs to make the decisions on the speed changing depends on the current data set of two sensors $s_{1}$ and $s_{2}$ in addition to the current walking speed. The construction of DTNNs is shown in Fig. 5. For the SFDTNNs the learning process through supervised training can be described as in (8) and (9).

$h(t)=\sigma\left(\left(s_{1}(t)-s_{2}(t)\right) * \omega_{i, j}^{1}(t)\right)+b_{i}^{h}$

$\lambda(t)=\eta\left(f\left(\sigma, \omega_{i, j}^{2}\right)\right)+b^{0}$

The special sigmoidal function is designed to allow larger numbers later, so it is varied in the specified rage $[0,1.6]$.

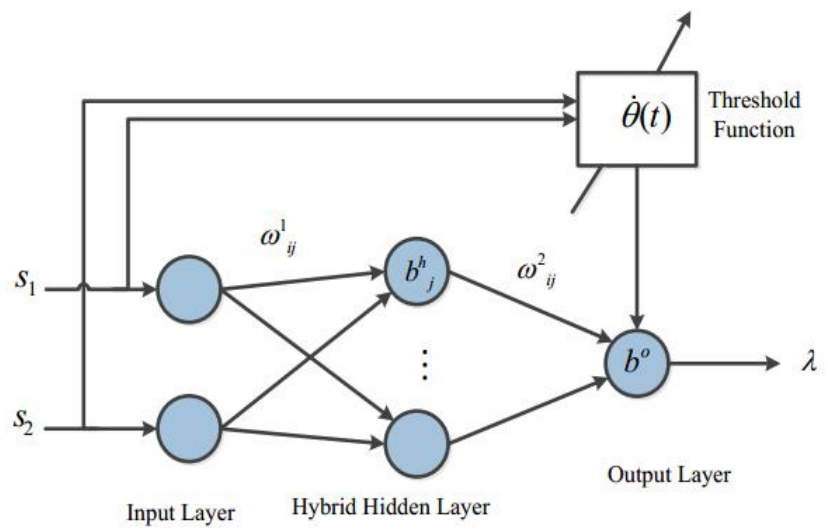

Fig.5. The proposed Dynamic Threshold Neural Networks (DTNNs) scheme. 
The changing of the walking speed during system navigation depends on the current walking speed for the pilot's safety, the minimum walking speed factor is 0.4 and the maximum is 1.6. According the mentioned above requirements, the accepted output of the SFNNs output due to the scaled activation function is:

1) $1.6 \geq \lambda(t) \succ 1$ for walking speed acceleration.

2) $\lambda(t)=1$ for no transition.

3) $1 \succ \lambda \geq 0.4$ for slowdown.

\section{B. Scaled sigmoidal training function}

The training process of the network is done by Back Propagation algorithm (BP). Before reviewing some of the variations and improvements which have been proposed to accelerate the learning process in neural networks, we briefly discuss the problems involved in trying to minimize the error function using gradient descent. Therefore, we first describe a simple modification of BP called BP with momentum rate, and then look at the form of the iteration path in weight space. The main steps in the calculations of BP training algorithm are described in the Table I. As networks input we take the difference between Heel off of the exoskeleton footboard and the pilot. The feed forward, back-propagation is the most popular training technique, effective and easy for multi-layered networks. The typical back propagation network has an input layer, an output layer, and at least one hidden layer as in our model. The training process normally uses some variant of the Delta Rule, which starts with the calculated difference between the actual output and the desired output. The complicated part of the training procedure is to determine which of the network inputs lead to the increase the output error, to be changed for correct output. During the network's training, the difference between the output of the output layer and the targeted output is back-propagated to the hidden layer for the connection weights adjustment using the Delta Rule. For the best combination of the learning rate $\eta$ and momentum $\lambda$ we experimentally identify the ideal value for the learning rate.

Non-standard Sigmoid Functions have been widely applied for many classification tasks, as non-linear tangential sigmoid [18], Log-Sigmoid
(LS) was demonstrated for the quick convergence [19] and parameterized Sigmoid for soft decisions [20]. We developed scaled-Sigmoid function for making soft decisions on walking speed transitions according to the experimentally identified thresholds and pilot intentions. Consider the permitted variation of the system's walking speed, the maximum limit factor is $\beta$ and the minimum one is $\alpha$ are take place to construct the proposed scaled-Sigmoid function.

$f(h)=\beta \frac{1}{1+e^{-h}}+\alpha$

where $\beta$ and $\alpha$ are learnable parameters depending on the current walking speed and maximum allowable one, which can make change to the function by scaling $f(h)$ linearly and shifting to the desired minimum value. Therefore, we associate the hidden layer activation function $f(h)$ with the parameters, $\beta$ and $\alpha$ we found that the scaling of the Sigmoid function with a factor will not affect the activation function performance. Fig. 6 illustrates the shape of the proposed scaled-Sigmoid function. The BP algorithm in which the weights of the network are updated immediately after the presentation each pair of input and target output is called the sequential learning. Training algorithms in order to perform gradient descent is formulated in stochastic form in which the network's weights modified on-line as in (11).

$\omega(t+\Delta t)=\omega(t)+\eta \delta(t) h(t)-\gamma \frac{\partial \delta(t)}{\partial \omega(t)}$

$\delta(t)=\lambda(t)-\lambda^{*}(t)$

where $\eta$ is the learning rate, $\delta(t)$ is the output error and $\lambda^{*}(t)$ denotes the targeted output. We chose supervised training process to find out the suitable weight set that minimizes the error between the real output and the target output. The procedure of the BP is to determine the error at the output layer then propagates backward through the hidden layer to the input layer to obtain the targeted output. We used the gradient descent to calculate the weights of the network and adjust them to minimize the output error. 


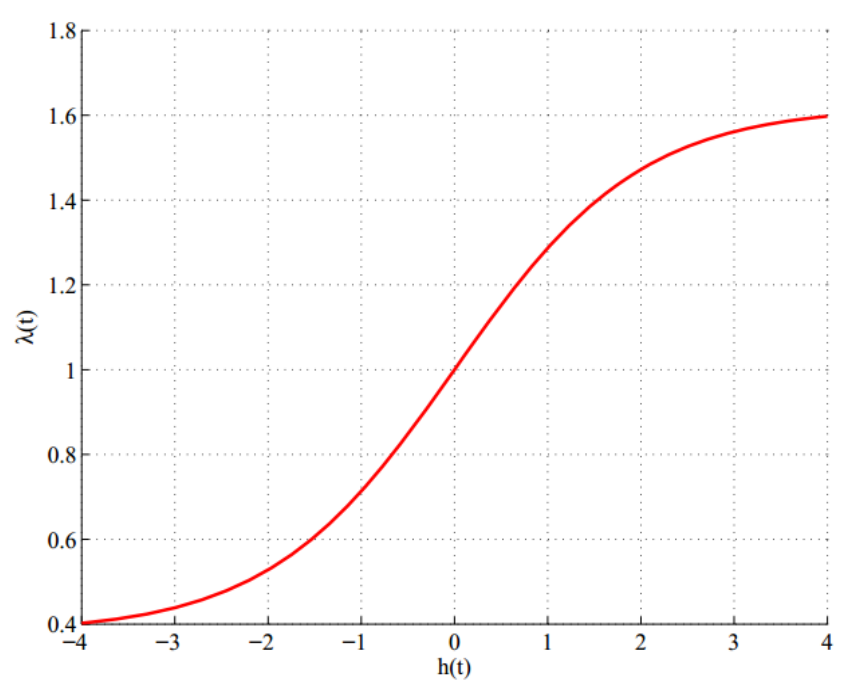

Fig.6. The proposed scaled-Sigmoid function.

TABLE I: SFNN TRAINING ALGORITHM

1. Initialize $\omega_{i, j}$ to small random values

2. Initialize $\lambda(0) \leftarrow 1$, tol. $=0.03, \eta=0.7$ and $\lambda=0.7$

3. Repeat

4. Get the input values $s_{1}(t), s_{2}(t)$ and training value $\lambda(t)$.

5. Calculate $\lambda(t)$ in a feed forward and Compare it with the thresholds.

6. Dependent on the current walking speed insure that the intended speed is allowable.

7. $\lambda \leftarrow \lambda(t)$.

8. Update $\omega_{i, j}$ through (11).

9. Until $\delta \prec$ tol. (tol. is a small positive number (error tolerance))

10. Return to step (4).

For the training procedure the heel contact time $H_{C T}$ of the pilot and the exoskeleton represent the main input for the network. From the experimental walking trails we found that, the $H_{C T}$ during flat walking ranges from $0.69 \pm 0.05$ and $0.41 \pm 0.07$ while walking speed varies between $1 \mathrm{~m} / \mathrm{s}$ and $2.5 \mathrm{~m} / \mathrm{s}$.

The input set consists of 50 pairs from the sensors $s_{1}$ and $s_{2}$ and 50 target output $\lambda$ experimentally calculated from speeds difference. The initial weight values and bias of the training algorithm are set to small random values. A learning rate $\eta=0.7$ and momentum rate $\gamma=0.7$ are used in $\mathrm{BP}$ algorithm for training process.

The response of the system to shadow the pilot's intentions for walking speed transitions is efficiently improved because of the new sensing technique preemptive prediction property. For the safety issues, we control $\lambda(t)$ according to the walking speed limitation to ensure that the pilot's intended speed is allowable. Every $\Delta(t)$ the network's output $\lambda(t)$ is first compared with intentions thresholds to decide the transition, then the intended walking speed is inspected to ensure the system's walking speed limitation. Here we mean that the algorithm must guarantee the transitions to be with in the given limitation.

\section{SIMULATION RESULTS AND EVALUATION}

\section{A. Data Collection}

For both walking and running, stance gets much shorter and swing gets slightly shorter as speed increases. As walking speed increases, the proportion of the cycle devoted to stance decreases and the proportion of the cycle devoted to swing increases. The $H_{C T}$ represents the period from heel strike to heel off every step. For the preemptive identification of the pilot's intentions for walking speed changing we just check the difference of heel off time, the heel off is the point at which the heel of the support leg leaves the ground. Because the heel off is not really a phase, it's just an instant in time event which properly can early identify the pilot's intentions for walking speed change. Knee and hip trajectories for the pilot and the exoskeleton are given with different speed changing intentions to find out the main SFNNs inputs. The heel off time was identified experimentally from the mutual heel off angle of the knee and the hip. The heel off time joints angles are just valid for the specified pilot (with a height of 181 $\mathrm{cm}$ and a weight of $70 \mathrm{~kg}$ ).

\section{B. 1-DOF exoskeleton platform simulations}

In order to show the efficiency of the proposed method, we compare its performance with the resulted performance of the previous work [21]. We developed DRF sensors system for walking speed changing intentions estimation for the HUALEX system. The last work performance drawback is that the decision on the intended walking speed takes the whole stance during one gait cycle. The previous work took the mismatch between exoskeleton and pilot to 
estimate the weighted parameter $\zeta$ calculated on-line from (13).

$\zeta=\frac{E x o_{C T}}{h_{C T}}$

But the proposed methodology gets the walking speed changing factor in the instant of heel of time, so it's a function of pilot's heel off time and exoskeleton's heel off time as in (14).

$$
\lambda=f\left(h_{\mathrm{HO}}, \mathrm{Exo}_{\mathrm{HO}}\right)
$$

We suppose that the initial system speed is $1.5 \mathrm{~m} / \mathrm{s}$, and the maximum allowed walking speed is $3 \mathrm{~m} / \mathrm{s}$ the initial weight values and biases are identified during learning process. The system parameters are estimated and corrected online through supervised learning algorithm by provide a set of inputs data for different interaction situation and its corresponding target outputs. The designed activation function according to the targeted output, sigmoid activation function for walking speed decisions. The block diagram of the proposed modification of VAC is illustrated in Fig. 7. The performance of proposed strategy is illustrated in Fig. 8.

\section{A. Performance Evaluation}

As depicted in Fig. 8 the interaction force between the exoskeleton and the pilot is shown for speed transition in the simulation experiment to show the efficiency of proposed strategy. For the gradual speed up the proposed walking speed control strategy works well, the resulting interaction force is significantly lower than the adaptive VAC strategy. The interaction force minimization resulted from the minimization of trajectory tracking error. The normalized mean squared error (nMSE) of angle trajectories in the proposed strategy is smaller than the adaptive VAC $\left(1.9^{\circ}\right.$ compared to $\left.2.41^{\circ}\right)$. The proper interaction force sensors of HUALEX make successfully investigation of admittance controller, therefore perfect angle correction. The practical measurements of joint angles, encoders

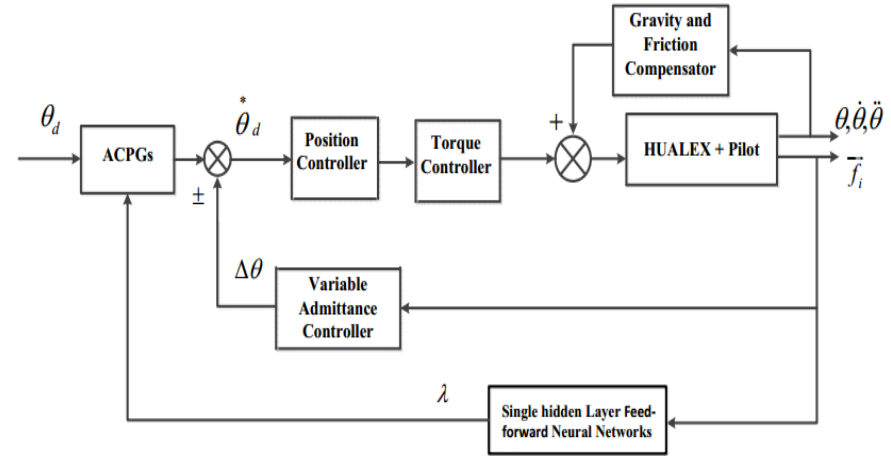

Fig. 7. The proposed Block Diagram of Walking Speed Control Strategy.

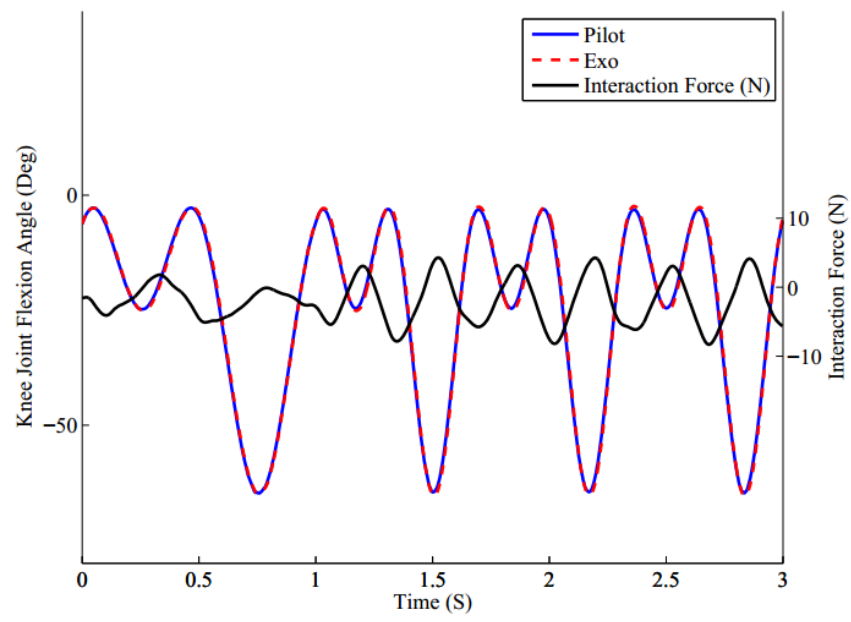

Fig. 8. The performance of NNs based Adaptive VAC for walking speed acceleration from $1 \mathrm{~m} / \mathrm{s}$ to $1.5 \mathrm{~m} / \mathrm{s}$.

on HUALEX and inclinometers on human limbs beside interaction forces between them are used to investigate the joint flexions for different stair height. Beside this reduced interaction force overshoot technique is applied. With high sensitivity of SAC, will lead to high cost control (complicated calculations and additional blocks) still the performance suffers from feedback overshoots according to pilot's intention. The separate treatment of the different gait phases (swing and stance) leads to modification in control efficiency and minimization in control cost in SAC and CAC. The comparison of the performance of the proposed modified VAC with the SAC and CAC for walking speed transition error is shown in the Fig. 9.

This will produce increased interaction forces in the contact places and worse performance of the control strategy.

As shown in Fig. 9 the performance efficiency of proposed strategy on gait transition control can be measured by the accuracy of tracking trajectory 


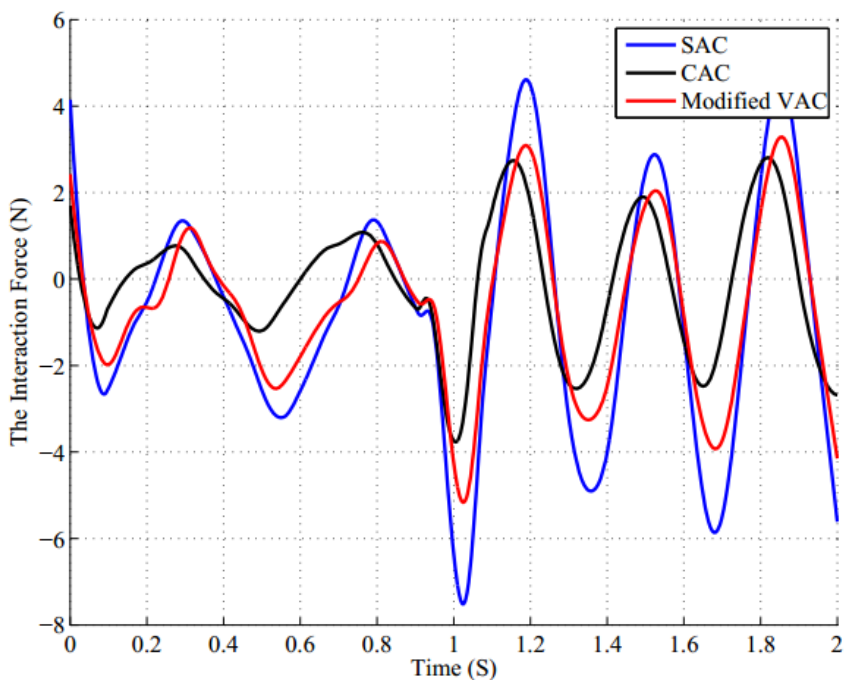

Fig. 9. Walking speed transition error.

during the transition and walking speed changing. The control algorithm minimizes the tracking error, as a natural result that transforming the interaction force to appropriate joint correction angle flexion.

\section{B. Performance Comparison}

The sudden changing in motion trajectories for dynamic obstacle avoidance during human-powered exoskeleton systems navigation needs special care additional efforts. As identified by computer simulations the proposed control strategy made good progress and smooth gait transitions between different modes.

Comparing to SAC, the proper interaction force sensors of HUALEX make successfully investigation of admittance controller, therefore perfect angle correction. The practical measurements of joint angles, encoders on HUALEX and inclinometers on human limbs beside interaction forces between them are used to investigate the joint flexions for different stair height. Beside this reduced interaction force overshoot technique is applied.

\section{CONCLUSIONS AND FUTURE WORK}

In this paper, we proposed a technique for preemptive identification of the pilot intentions to predict the next step of the human-exoskeleton system. Our control method minimized the tracking error during gait transition for human-exoskeleton system by reducing the interaction force overshoot.
On the basis of the networks input data (sensors output), we able to predict the intended walking speed and also compensate the future interaction between the pilot and the exoskeleton. This preemptive compensation of intended interaction leads to big betterment in the motion control performance. In the future work will focus on the system response optimization to minimize the transition error, and transition time towards form brain to muscles, then HUALEX can moves freely for more complicated missions. Also we're planning to work in obstacle avoidance in general and adapt to difference stairs height and tread length this will overcome the proposed technique limitations, then will go forward for practical implementation.

\section{REFERENCES}

[1] Ka M, Cheng $\mathrm{H}$, Toan $\mathrm{T}$ H, Jing Q. “Minimizing Human-Exoskeleton Interaction Force Using Compensation for Dynamic Uncertainty Error with Adaptive RBF Network" , Journal of Intelligent and Robotic Systems, 2015, 82: 413--433.

[2] H. Kazerooni, A. Chu, R. Steger, "That Which Does Not Stabilize, Will Only Make Us Stronger", International Journal of Robotics Research, vol. 26, 2007, pp. 75-89.

[3] A. Zoss , H. Kazerooni, A. Chu, "On the Mechanical Design of the Berkeley Lower Extremity Exoskeleton (BLEEX)",International Conference on Intelligent Robots and Systems, Edmenton, Canada, 2005, pp. 3132-3139.

[4] A. I. A. Ahmed, H. Cheng, Z. Liangwei, M. Omer, X. Lin, “On-line Walking Speed Control in Human-Powered Exoskeleton Systems Based on Dual Reaction Force Sensors", Journal of Intelligent and Robotic Systems, vol. 87 , no.1 , 2017, pp. 59-80.

[5] H. Kazerooni, J. L. Racine, L. Huang, R. Steger, "On the Control of the Berkeley Lower Extremity Exoskeleton (BLEEX)", International Conference of Robotics and Automation, Barcelona, Spain, 2005, pp. 4353-4360.

[6] J. Ghan, R. Steger, H. Kazerooni, “Control and System Identification for the Berkeley Lower Extremity Exoskeleton", Advanced Robotics, vol. 20, 2006, pp. 989-1014.

[7] Y. Sankai, "HAL: Hybrid Assistive Limb based on Cybernics", Robotics Research, 2011, pp. 25-34.

[8] S. Lee, Y. Sankai, "Virtual Impedance Adjustment in Unconstrained Motion for an Exoskeletal Robot Assisting the Lower Limb", Advanced Robotics, vol. 19, 2005, pp. 73-795.

Okunev V., Nierhoff T., H.S.: Human-preference-based control design : Adaptive robot admittance control for physical human-robot interaction. In: The 21st IEEE International Symposium on Robot and Human Interactive Communication, 2012, pp. 443-448.

[9] Kim J W, Jang H J, Hwang D H, Park C., "A Step, Stride and Heading Determination for the Pedestrian Navigation System", Journal of Global Positioning Systems, vol. 3, 2004, pp. 273-279.

[10] Righetti L, Buchli J, Ijspeert A J, “Dynamic hebbian learning in adaptive frequency oscillators" Physica D: Nonlinear Phenomena, vol. 216, 2005, pp. 269-281.

[11] Isakov E, Burger H, Krajnik J, Grgoric M, Marincek C., "Influence of speed on gait parameters and on symmetry in transtibial amputees", 
Prosthetics and Orthotics International, vol. 20, 1996, pp. 153-158.

[12] Mondal S, Nandy A, Verma C, Shukla S, Saxena N, Chakraborty P, Nandi G C., "Modeling a Central Pattern Generator to Generate the Biped Locomotion of a Bipedal Robot Using Rayleigh Oscillators", Communications in Computer and Information Science, vol. 168, 2011, pp. 289-300.

[13] Hornik K, Stinchcombe M, White H., "Multilayer feedforward networks are universal approximators", Neural Networks, 1989, vol.2, pp. 359-366.

[14] Chen T P, Chen H., “Universal approximation to nonlinear operators by neural networks with arbitrary activation functions and its application to dynamical systems", IEEE Transactions on Neural Networks, 1995, vol. 6, pp. 911-917.

[15] S. Lee, Y. Sankai, "Power Assist Control for Walking Aid with HAL-3 Based on EMG and Impedance Adjustment around Knee Joint", International Conference on Intelligent Robots and Systems, EPFL, Switzerland, 2002, pp. 1499-1504.

[16] A. I. A. Ahmed, H. Cheng, X. Lin, Z. Elhassan, M. Omer, "On-line Walking Speed Control in Human-Powered Exoskeleton Systems", IEEE International Conference on Communication, Control, Computing and Electronic Engineering(ICCCCEE2017), Khartoum, Sudan, 2017, pp.1-7.

[17] Tanvi S, Arpit A, Annu B, Mahesh C.,"SVM and ANN: A Comparative Evaluation", 1st International Conference on Next Generation Computing Technologies (NGCT-2015), Dehradun, Uttrakhand, India, 2015, pp. 960-964.

[18] Roman A P., "Log-Sigmoid Multipliers Method in Constrained Optimization", Annals of Operations Research, 2001, vol. 101, pp. 427-460.

[19] Zhang C, Woodland P C., "Parameterised Sigmoid and ReLU Hidden Activation Functions for DNN Acoustic Modelling", IEEE International Conference on Acoustics, Speech and Signal Processing (ICASSP), Shanghai, China, 2016, pp. 5300-5304.

[20] Abusabah I. A. Ahmed, H. Cheng, A. R. Abdalla and G. Hongliang, "Modified admittance control for maneuverable human-powered augmentation lower exoskeleton systems", $20172^{\text {nd }}$ International Conference on Advanced Robotics and Mechatronics (ICARM), Hefei, 2017, pp. 7-12.

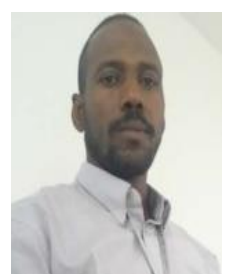

Abusabah I. A. Ahmed is assistant professor at the Department of Electrical Engineering, Karary University. He received the M.Sc. degree and Ph. D. degree in Electronic Engineering from University of Electronic Science and Technology of China in 2013 and 2017. His research area includes Robotics Control, Predictive and Adaptive Control and Neural Networks. Dr Abusabah has 6 academic publications in international journals and conferences in Robotics area. He is a reviewers for many journals and conferences (JIRA, International Journal of Intelligent Robotics and Application $s$,ICCCCEE). Now he is teaching Neural networks and Embedded Systems design for graduate students, and Control Theory, Automatic Control, Modern Control, Optimal Control and Electronic Measurements for junior students at Karary University.

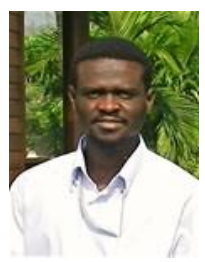

Mohammed H. H. Musa (1981), was born in Kadugli, Sudan, on May 29, 1981. He received a B.Sc. and M.Sc. degree in Electrical Engineering from Sudan University of Science and Technology in 2005 and 2010. He received a Ph.D. degree from Southwest Jiaotong University Nov 2018. He worked in National Electricity Corporation (NEC), Sudan 2006-2010. Presently, he work as assistant professor in college of engineering at Almughtarbeen University, and as senior technical support at Sudanese Thermal Power Generating Company (STPG) up to now. 